\title{
AVALIAÇÃO DOS METABÓLITOS PRODUZIDOS POR FOTOFERMENTAÇÃO POR CIANOBACTÉRIAS
}

\author{
L.P. VAZ ${ }^{1}$, J. S. FERREIRA ${ }^{2}$ \\ ${ }^{1}$ Universidade Federal de Uberlândia, Graduação em Biotecnologia \\ 2 Universidade Federal de Uberlândia, Professora da FEQUI/UFU \\ E-mail para contato: luisapiresvaz@gmail.com
}

\begin{abstract}
RESUMO - As cianobactérias são microrganismos procariontes com grande impacto sobre o ciclo de carbono, reciclagem do oxigênio global e de fixação do nitrogênio. Elas têm sido amplamente estudadas pelo seu potencial de fixar o $\mathrm{CO}_{2}$, com energia obtida fotossinteticamente, para síntese de produtos de alto valor agregado, como, compostos antimicrobianos, antivirais e anticancerígenos, toxinas, fitohormônios, biocombustíveis de terceira geração e ácidos orgânicos. A proposta deste trabalho foi identificar os possíveis metabólitos (etanol e ácidos orgânicos) sintetizados pelas cianobactérias Nostoc sp PCC 7423 e Anabaena variabilis ATCC 29413, por fotofermentação. Foi avaliado a influência do enriquecimento do meio $\mathrm{BG} 11_{0}$, usando glicose como fonte de carbono orgânico, em fotoperíodo de $12 \mathrm{~h}$ a $30^{\circ} \mathrm{C}$ por até 7 dias de processo. Verificou-se que houve a formação de ácido acético por $A$. variabilis em meio $\mathrm{BG} 11_{0}$ e produção de etanol por Nostoc sp em meio enriquecido com glicose.
\end{abstract}

\section{INTRODUÇÃO}

As cianobactérias são organismos procariontes, fotoautotróficos, gram-negativas, podendo ser filamentosas ou unicelulares e estão entre as formas de vida mais bem sucedidas e antigas ainda presentes na Terra (GADEMANN e PORTMANN, 2008). Graças a adaptações bioquímicas, fisiológicas, genéticas e reprodutivas estes organismos foram capazes de colonizar diversos tipos de ecossistemas terrestres e aquáticos. Sua longa história evolutiva proporcionou grande diversidade morfológica e fisiológica, incluindo a produção de metabólitos secundários, os quais despertam interesse comercial por possuírem atividades biológicas importantes (BAJPAI, 2013). Entretanto, o único processo economicamente viável realizado atualmente é o do cultivo das cianobactérias em tanques abertos, visando o uso em suplementação alimentar de animais, extração de pigmentos, além de biomassa para produção de biocombustíveis (HALLENBECK, 2012).

Em relação aos metabólitos obtidos por esses microrganismos, pode-se citar compostos bioativos, que demonstram atividades antitumorais, antivirais, antibacteriana, antifúngica, antimalárica, antimicóticas, antiproliferativos e agentes imunossupressores e que, devido à sua elevada estabilidade química e solubilidade em água, tem aplicações importantes em indústrias alimentícias, farmacêuticas e de cosméticos (TAN, 2007). Além disso, podem secretar enzimas exploradas comercialmente, tais como a beta-lactamase e a lipase (PRABHAKARAN et al., 1994) e ainda, são capazes de sintetizar biopolímeros, tais como o 
PHB (poli- $\beta$ - hidroxibutirato), um polímero em potencial para a produção de bioplástico por apresentar características de biodegradabilidade, termoplasticidade, biocompatibilidade com células e tecidos humanos, possível aplicação para a produção de nanofibras, além de ser proveniente de fonte renovável (SHARMA, 2005).

As cianobactérias também são capazes de sintetizar ácidos orgânicos, dentre os quais se encontra o ácido lático, um produto de grande interesse econômico, sendo este muito utilizado na indústria alimentícia como acidulante e flavorizante e para a produção de polímeros biodegradáveis. Entretanto, os ácidos orgânicos não são sintetizados em grande quantidade pelas cianobactérias, devido à utilização do carbono fixado para a manutenção celular (ANGERMAYR et al., 2014). Com relação aos biocombustíveis, que são uma das alternativas para suprir a escassez futura de recursos fósseis, pois são advindos de fontes renováveis e apresentam baixos custos de produção, o etanol pode ser sintetizado pelas cianobactérias, o qual é um subproduto do ciclo do Calvin (HALLENBECK, 2012).

Uma das formas de induzir a produção de metabólitos secundários é pelo processo fermentativo, ou seja, garantir a anaerobiose do meio para que a rota metabólica do microrganismo seja alterada, e desta forma haja a produção do ácido de interesse. Diante deste contexto, a proposta deste trabalho foi avaliar o potencial de produção de diferentes metabólitos por fotofermentação de duas espécies de cianobactérias: Anabaena variabilis ATCC 29413 e Nostoc sp. PCC 7423. Foi analisada a influência da composição do meio de cultivo, empregando meio BG11 $1_{0}$ (sintético) como meio autotrófico e meio enriquecido com glicose, usada como fonte de carbono orgânico.

\section{MATERIAIS E MÉTODOS}

\subsection{Microrganismo e Condições de Cultivo}

As cepas de cianobactérias utilizadas, Nostoc sp PCC 7423 e Anabaena variabilis ATCC 29413, foram cedidas pela Unidade de Pesquisa em Cianobactérias da FURG (RS). Elas foram cultivadas em meio BG11 $1_{0}$ (RIPPKA et al., 1979). O cultivo das cianobactérias foi feito em uma câmara com fotoperíodo de $12 \mathrm{~h}$ e intensidade luminosa de 1800 lux a $30^{\circ} \mathrm{C}$.

\subsection{Preparação dos Frascos para Fermentação}

Para realizar a fermentação, toda a vidraria (pipeta, funis, frascos de penicilina, Erlenmeyers) e meio utilizados foram previamente esterilizados em autoclave a $121^{\circ} \mathrm{C}$ por 20 min, a fim de evitar uma possível contaminação. Os frascos foram inoculados em câmara de fluxo laminar esterilizada com álcool $70 \%$ e luz ultravioleta por $15 \mathrm{~min}$. Os ensaios de fotofermentação foram realizados em batelada usando frascos de penicilina de $15 \mathrm{~mL}$ de volume útil, sendo $1,125 \mathrm{~mL}$ o volume do inóculo. Como o intuito do trabalho foi avaliar o desempenho das duas espécies para as diferentes condições de meio, foram preparados dois

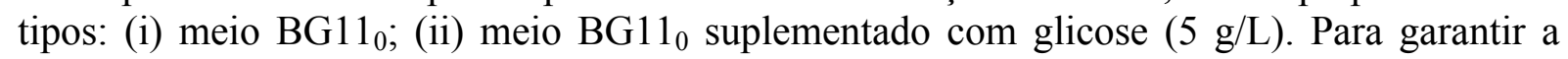


anaerobiose do meio, foi borbulhado gás argônio (Ar; 99,999\%) durante 3 minutos em cada frasco. Em seguida, os frascos foram selados com septos de borracha butílica e lacrados com uma cápsula de alumínio. Seringas de $5 \mathrm{~mL}$ graduadas foram colocadas como válvula de escape, caso ocorresse a produção de algum biogás. Frascos de sacrifícios foram utilizados permitir a análise da composição do meio fermentativo e de células para intervalos diferentes de tempo (Figura 1). As análises foram feitas no terceiro e no sétimo dia de fotofermentação e em triplicata.

Figura 1 - Fotofermetação por cianobactérias em batelada usando frascos de penicilina.

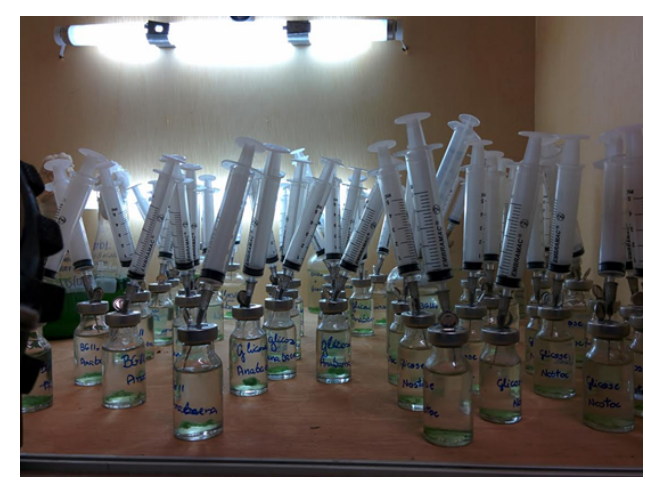

\subsection{Determinação da concentração celular}

A determinação da concentração celular inicial e final foi realizada através do método gravimétrico, correlacionando a medida da absorbância do inóculo dos microrganismos através de um espectrofotômetro (GENESYS) e a massa de sólidos voláteis (SV) (BERBEROĞLU et al., 2008). A densidade óptica a $647 \mathrm{~nm}\left(\mathrm{OD}_{647}\right)$ foi medida em um espectrofotômetro, e para cada cepa foram obtidas duas curvas de calibração, uma para cada comprimento de onda.

\subsection{Análise das amostras}

O meio de fermentação foi analisado para a determinação de glicose consumida e metabólitos (ácidos orgânicos e etanol) formados. Utilizou-se o método de cromatografia liquida de alta performance (HPLC) realizada no cromatógrafo marca Shimadzu modelo LC20A Prominence, coluna SUPELCOGEL C-610H. Empregou-se como fase móvel ácido fosfórico $(0,1 \%)$, operou-se com fluxo de $0,5 \mathrm{~mL} / \mathrm{min}$, temperatura do forno $32^{\circ} \mathrm{C}$ e volume de injeção de $20 \mu \mathrm{L}$.

\section{RESULTADOS E DISCUSSÃO}

As cianobactérias são microrganismos fotossintetizantes, mas que também podem se desenvolver em meios anóxicos, resultantes do cultivo na ausência de luz. A capacidade de cianobactérias empregarem rotas metabólicas alternativas na condição de meios anaeróbios e no escuro, pode levar à síntese de produtos de valor comercial por fermentação, como $\mathrm{CO}_{2}$, $\mathrm{H}_{2}$, ácidos acético, fórmico, láctico e etanol (STAL e MOEZELAAR, 1997). Neste estudo, 
investigou-se os possíveis metabólitos que podem ser formados por fermentação pelas cianobactérias Nostoc sp PCC 7423 e Anabaena variabilis ATCC 29413, em meio BG11 enriquecido ou não com glicose $(5 \mathrm{~g} / \mathrm{L})$, sob anaerobiose e em fotoperíodo de $12 \mathrm{~h}$. Os resultados de concentração de glicose consumida e dos metabólitos identificados são apresentados na Tabela 1 e o perfil de concentração celular por 7 dias está ilustrado na Figura 2 .

Tabela 1: Dados da fermentação por Nostoc sp PCC 7423 e Anabaena variabilis ATCC 29413 durante 7 dias, em meio BG11 ${ }_{0}$ sem e com glicose, sob fotoperíodo de $12 \mathrm{~h}$.

\begin{tabular}{|c|c|c|c|c|c|}
\hline Cepa & $\begin{array}{c}\text { Composição do } \\
\text { meio } \\
\end{array}$ & $\begin{array}{l}\text { Tempo } \\
\text { (dias) }\end{array}$ & $\begin{array}{c}C_{\text {glicose }} \\
(\mathrm{g} / \mathrm{L})\end{array}$ & $\begin{array}{l}\mathrm{C}_{\text {etanol }} \\
(\mathrm{g} / \mathrm{L})\end{array}$ & $\begin{array}{c}C_{\text {ácido acético }} \\
(\mathrm{g} / \mathrm{L})\end{array}$ \\
\hline \multirow{6}{*}{$\begin{array}{c}\text { Anabaena } \\
\text { variabilis }\end{array}$} & \multirow{3}{*}{$\mathrm{BG} 11_{0}$} & 0 & --- & --- & -- \\
\hline & & 3 & --- & --- & 0,25 \\
\hline & & 7 & ---- & --- & --- \\
\hline & BG11 0 & 0 & 5,00 & --- & --- \\
\hline & + & 3 & 0,61 & --- & --- \\
\hline & glicose & 7 & 0,44 & --- & --- \\
\hline \multirow{6}{*}{ Nostoc sp } & \multirow{3}{*}{$\mathrm{BG} 11_{0}$} & 0 & ---- & --- & --- \\
\hline & & 3 & ---- & --- & --- \\
\hline & & 7 & ---- & --- & --- \\
\hline & BG11 0 & 0 & 5,00 & --- & --- \\
\hline & + & 3 & 1,46 & 0,12 & --- \\
\hline & glicose & 7 & 0,90 & 0,20 & --- \\
\hline
\end{tabular}

Sob as condições de fermentação, verificou-se que apenas a cepa Nostoc sp produziu etanol em meio enriquecido com glicose, sendo que a concentração foi de $0,12 \mathrm{~g} / \mathrm{L}$ e $0,20 \mathrm{~g} / \mathrm{L}$ para 3 dias e 7 dias de fermentação, respectivamente. Portanto, verificou-se que a condição de meio heterotrófico favoreceu a produção do etanol para esta cepa. O segundo metabólito identificado foi o ácido acético. No entanto, houve a produção de $0,25 \mathrm{~g} / \mathrm{L}$ deste composto somente pela Anabaena variabilis em meio BG11 $1_{0}$ sem glicose.

De acordo com o estudo realizado por Moezelaar e Stal (1997), a cianobactéria Microcystis PCC7806, produziu quantidades equimolares de ácido acético e etanol ao fermentar o glicogênio armazenado, em meio anaeróbico e no escuro. Quando as cepas foram cultivadas em fotoperíodo de $16 \mathrm{~h}$ de luz e $8 \mathrm{~h}$ no escuro, houve uma produção de etanol. A influência do enriquecimento com fonte de carbono orgânico também foi empregada por Costa et al. (2015) para a produção de etanol por fotofermentação pela alga verde Chlamydomas reinhardtii. Neste trabalho, foi alcançada uma produção de 19,25 g/L de etanol em meio TAP com depleção de enxofre e sem adição de lactose ao meio. Além do etanol, também foram produzidos os ácidos propiônico e acético.

Os dados obtidos neste trabalho indicaram que as cepas avaliadas Anabaena variabilis e Nostoc sp podem produzir ácido acético e etanol, dependendo das condições de fermentação. Entretanto, faz-se necessário ampliar o número de parâmetros a serem investigados no intuito de compreender a influência das condições de processo que levam a alteração da rota metabólica destes microrganismos, induzindo a formação de ácidos orgânicos e etanol. Portanto, outras variáveis como fotoperíodo, intensidade luminosa, temperatura, concentração 


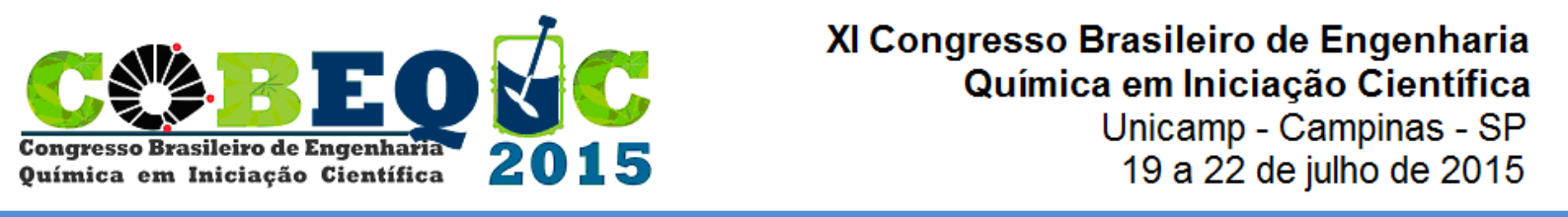

de célula e de fonte de carbono, tipo de fonte de carbono e tempo de fermentação devem ser estudadas.

Com relação ao crescimento celular, foi verificado que o meio heterotrófico induziu o aumento da biomassa, sendo que a concentração de células sofreu um acréscimo de 2 vezes para Anabaena variabilis e de 2,6 para Nostoc sp, em 7 dias. Principalmente no caso da Anabaena variabilis, houve consumo de glicose somente para crescimento celular sem produção de metabólitos como ocorreu no ensaio empregando a Nostoc sp. Para o meio BG11 $1_{0}$, este aumento foi de 1,7 vezes para a Nostoc $\mathrm{sp}$, variando de $0,12 \mathrm{~g} / \mathrm{L}$ para $0,21 \mathrm{~g} / \mathrm{L}$ ao final do ensaio. Entretanto, para a Anabaena variabilis, foi verificado um aumento da concentração celular de $0,18 \mathrm{~g} / \mathrm{L}$ para $0,19 \mathrm{~g} / \mathrm{L}$ nos 3 primeiros dias de ensaio e, posteriormente, a concentração de célula foi de $0,15 \mathrm{~g} / \mathrm{L}$ em 7 dias de fermentação.

Figura 2 - Variação de concentração celular em função do tempo.

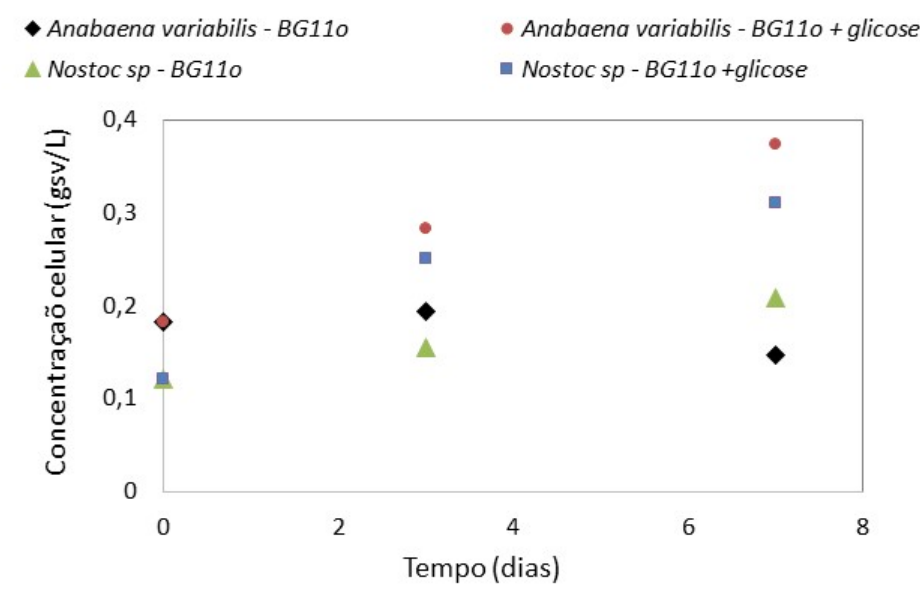

\section{CONCLUSÕES}

As cianobactérias asseguram um futuro promissor devido às inúmeras possibilidades de metabólitos ainda não explorados, seu fácil cultivo e baixo custo de manutenção. Este trabalho identificou a produção de ácido acético por Anabaena variabilis em meio autotrófico e de etanol em meio enriquecido com glicose por Nostoc sp. O estudo demostra que a exploração de parâmetros de cultivo podem levar à compreensão do como as condições de fotofermentação podem influenciar as rotas metabólicas das cianobactérias e aumentar a produção destes compostos.

\section{AGRADECIMENTOS}

Os autores agradecem a Universidade Federal de Uberlândia e a Faculdade de Engenharia Química pela oportunidade em realizar este trabalho, à Unidade de Pesquisa em Cianobactérias da FURG (RS) pela doação das cepas e ao apoio financeiro da FAPEMIG, do CNPq e da CAPES. 


\section{REFERÊNCIAS}

ANGERMAYR, S. A., et al. Exploring metabolic engineering design principles for the photosynthetic production of lactic acid by Synechocystis sp. PCC6803. Biotechnology for Biofuels.v. 7:99, p.1-15, 2014.

BAJPAI, R.D.; SUSEELA, M.R. Cyanobacteria: potencial candidates for drug discovery. Springer. v. 103, p.947-961, 2013.

BERBEROĞLU, H, JAY, J., PILON, L. Effect of nutrient media on photobiological hydrogen production by Anabaena variabilis ATCC 29413. International Journal Of Hydrogen Energy. v. 33, p. 1172-1184, 2008.

COSTA, R.L, OLIVEIRA, T.V., FERREIRA, J.S , CARDOSO, V.L., BATISTA, F.R.X. Prospective technology on bioethanol production from photofermentation. Bioresource Technology. v.181, p. 330-337, 2015.

GADEMANN, K.; PORTMANN, C. Secondary metabolites from cyanobacteria: complex structures and powerful bioactivities. Curr Org Chem . v. 12, p. 326-341, 2008.

HALLENBECK, P. C. Hydrogen Production by Cyanobacteria. Microbial Technologies in Advanced Biofuels Production.p. 15-28, 2012.

MOEZELAAR, R., STAL, L.J. A comparison of fermentation in the cyanobacterium Microcystis PCC7806 grown under a light/dark cycle and continuous light. European Journal of Phycology.v. 32, p. 373-378, 1997.

PRABHAKARAN, D.; SUMATHI, M.; SUBRAMANIAN, G. Ability to use amphicillin as nitrogen source by marine cyanobacterium Phormidium valderianum BDU 30501. Curr. Microbiol. v. 28, p, 315-320, 1994.

RIPPKA R, DESRUELLES J, WATERBURY JB, HERDMAN M, STANIER RY. Generic assignments, strain histories and properties of pure cultures of cyanobacteria. $J$ Gen Microbiol. v. 111, p. 1-61, 1979.

SHARMA, L.; MALLICK, N. Accumulationof poly- $\beta$-hydroxybutyrate in Nostoc muscorum: regulation $\mathrm{pH}$, light-dark cycles, $\mathrm{N}$ and $\mathrm{P}$ status abd carbon sources. Bioresource Technology, v. 96, p. 1304-1310, 2005.

STAL, L.J., MOEZELAAR, R. Fermentation in cyanobacteria. FEMS Microbiology Reviews. v. 21, p. 179-211, 1997.

TAN, L.T. Bioactive natural products from marine cyanobacteria for drug discovery. Phytochemistry. v. 68, p. 954-979, 2007. 